Service social

\title{
Documenter l'émergence de la militance autour des enjeux concernant les jeunes LGBTQ au Québec
}

\section{Olivia Kamgain, Line Chamberland et Joseph J. Lévy}

Volume 63, numéro 2, 2017

Genres et sexualités chez les jeunes

URI : https://id.erudit.org/iderudit/1046503ar

DOI : https://doi.org/10.7202/1046503ar

Aller au sommaire du numéro

\section{Éditeur(s)}

École de travail social et de criminologie de l’Université Laval

ISSN

1708-1734 (numérique)

Découvrir la revue

Citer cet article

Kamgain, O., Chamberland, L. \& Lévy, J. J. (2017). Documenter l'émergence de la militance autour des enjeux concernant les jeunes LGBTQ au Québec. Service social, 63(2), 114-131. https://doi.org/10.7202/1046503ar
Résumé de l'article

Cet article documente l'émergence de la militance autour des enjeux sociaux concernant les jeunes LGBTQ, en analysant les récits de huit militants ayant eu une implication communautaire en lien avec ces enjeux au cours des années 1990 et 2000. Les principaux enjeux soulevés sont les expériences de victimisation vécues par les jeunes et l'absence de ressources à leur intention. Les stratégies d'action s'orientent vers la mise en place d'organismes par et pour les jeunes et de regroupements des organisations jeunesse LGBTQ. Elles comprennent aussi des interventions de sensibilisation dans les écoles et des actions directes associant l'art et la performance. Ces militants font un bilan positif de leurs actions. Les organisations constituées autour des enjeux touchant les jeunes LGBTQ ont su établir leur légitimité et développer des collaborations avec des partenaires communautaires, institutionnels et gouvernementaux. 


\title{
Documenter l'émergence de la militance autour des enjeux concernant les jeunes LGBTQ au Québec
}

\author{
KAMGAIN, Olivia, M.A., administration publique, adjointe de recherche \\ CHAMBERLAND, Line, Ph. D. sociologie, professeure au département de sexologie \\ LÉVY, Joseph J., Ph. D. anthropologie, professeur associé au département de sexologie
}

\section{RÉSUMÉ}

Cet article documente l'émergence de la militance autour des enjeux sociaux concernant les jeunes LGBTQ, en analysant les récits de huit militants ayant eu une implication communautaire en lien avec ces enjeux au cours des années 1990 et 2000. Les principaux enjeux soulevés sont les expériences de victimisation vécues par les jeunes et l'absence de ressources à leur intention. Les stratégies d'action s'orientent vers la mise en place d'organismes par et pour les jeunes et de regroupements des organisations jeunesse LGBTQ. Elles comprennent aussi des interventions de sensibilisation dans les écoles et des actions directes associant l'art et la performance. Ces militants font un bilan positif de leurs actions. Les organisations constituées autour des enjeux touchant les jeunes LGBTQ ont su établir leur légitimité et développer des collaborations avec des partenaires communautaires, institutionnels et gouvernementaux.

Mots-clés : Jeunes LGBTQ, mouvement communautaire, mouvement LGBT, Québec

\begin{abstract}
This article documents the emergence of social activism by and for LGBTQ youth by analyzing the stories of eight community activists involved in these issues during the 1990s and 2000s. Victimization experienced by youth and the lack of resources dedicated to them were the main issues. The strategies of action are directed towards the establishment of organizations by and for young people and the gathering of LGBTQ youth organizations. They also include awareness raising activities in schools and direct actions combining art and performance. These activists make a positive assessment of their actions. Organizations formed around issues affecting LGBTQ youth have been able to establish their legitimacy and develop collaborations with community, institutional and government partners.
\end{abstract}

Key words: queer youth, community-based movement, LGBT movement, Quebec 


\section{INTRODUCTION}

Au Québec et au Canada, les dernières décennies ont été marquées par des avancées considérables en matière de droits pour les personnes lesbiennes, gaies et bisexuelles et, plus récemment, pour les personnes trans, grâce aux mobilisations de plusieurs militant.e.s depuis les années 1960. Avant les années 1990, la génération pionnière se mobilise principalement contre les violences, policières et quotidiennes, et contre les discriminations envers les gais et les lesbiennes dans toutes les sphères de vie. Entre 1990 et 2005, une seconde génération d'activistes milite pour l'acquisition de droits, avec pour objectif l'atteinte de l'égalité juridique pour les couples de même sexe et pour les familles homoparentales (Demczuk et Remiggi, 1998 ; Chamberland et al., 2018). Malgré les convictions que les inégalités seraient désormais corrigées, les personnes lesbiennes, gaies, bisexuelles, trans et queers (LGBTQ) ont continué de vivre diverses formes de discrimination et rencontrent des obstacles similaires à ceux des générations précédentes, tels que des attitudes de rejet, des violences physiques et verbales et, conséquemment, la peur de faire son coming out. Dans ce contexte ambivalent, de nouveaux acteurs collectifs, prenant conscience des injustices et des discriminations qui touchent les jeunes LGBTQ (J-LGBTQ), les personnes trans et les personnes LGBTQ provenant des communautés ethnoculturelles, se regroupent et développent un « agir-ensemble intentionnel » (Neveu, 2015, p. 9) sous la forme d'un large registre d'actions pour faire valoir leurs revendications tant au sein du mouvement communautaire LGBTQ qu'auprès des décideurs publics (Chamberland et al., 2018). En ce qui concerne les jeunes, on dénote l'émergence dans le discours politique d'enjeux comme l'intimidation dans le milieu scolaire et le manque de ressources d'accueil et de soutien qui tiennent compte des spécificités de leurs situations. Dans la perspective ouverte par ces travaux, cet article vise à documenter, à partir des récits d'activistes impliqués dans les luttes pour l'amélioration des conditions de vie des J-LGBTQ pendant les décennies 1990 et 2000, les réalités et les enjeux auxquels ceux-ci étaient confrontés, les stratégies d'action privilégiées et leurs réflexions quant au bilan de ces actions.

\section{MÉTHOdOLOGIE}

Les données proviennent d'un corpus de témoignages, livrés hors de l'anonymat (sauf exception), constitué dans le cadre de la recherche InterReconnaissance. Mémoire, droits et reconnaissance du mouvement communautaire au Québec (sous la direction de Francine Saillant ${ }^{1}$ ). Ce projet visait à retracer la mémoire des luttes menées pour les droits et l'égalité au Québec par le biais d'entretiens semi-dirigés auprès de personnes y ayant non seulement participé, mais aussi exercé un leadership reconnu, selon l'avis de comités consultatifs de pairs, dans cinq secteurs d'action : femmes, handicap, immigration, santé mentale et LGBTQ. Pour ce dernier secteur, des entretiens ont été réalisés auprès de 37 acteur.trice.s-témoins représentant trois cohortes d'activistes : des leaders impliqués dans les luttes pionnières d'avant les années 1990 ; des leaders ayant combattu pour la reconnaissance et l'égalité juridique entre 1990 et 2005 et finalement, des personnes dont le leadership s'est affirmé dans les années 2000 autour d'enjeux du nouveau millénaire. Les entretiens portaient sur leur parcours personnel, leur engagement dans la structuration du mouvement LGBT, les luttes menées, le répertoire et le bilan des actions menées. D'une durée de 1 heure à 2 heures et demie, ils ont eu lieu entre février 2013 et octobre 2014 puis ont été codifiés à l'aide du logiciel NVivo en tenant compte des thèmes

\footnotetext{
${ }^{1}$ Cette recherche a bénéficié du soutien financier du Conseil de recherches en sciences humaines du Canada. Elle regroupait des chercheur.e.s de l'Université Laval, de l'Université de Montréal et de l'Université du Québec à Montréal (UQAM).
} 
majeurs évoqués dans les entretiens. Le projet a reçu l'approbation éthique des trois universités de rattachement de l'équipe de chercheur.e.s.

Dans le cadre du présent article, nous avons retenu les témoignages de leaders ayant eu un engagement communautaire dans des organismes jeunesse LGBTQ ou intervenant en relation avec des enjeux jeunesse, soit un sous-groupe de huit participants (six hommes cis se définissant comme gais et deux hommes s'identifiant comme queers ou non binaires au moment de l'entrevue ${ }^{2}$ ). Deux appartiennent à la seconde cohorte et situent le début de leur activisme dans la période des années 1990 et six appartiennent à la troisième cohorte. La période que recouvre leur militantisme s'étend donc de 1990 à 2014, mais c'est surtout dans les années 2000 que les organismes jeunesse LGBTQ acquièrent une visibilité sociale en se regroupant. Quatre participants ont milité dans des organismes régionaux hors de Montréal (Gatineau, Québec, Lanaudière et Bas-du-Fleuve), pendant la totalité ou une partie de leur parcours d'activisme. Un participant sur deux a contribué à la mise sur pied d'un regroupement régional ou national, parallèlement à son implication locale. Dans cet article, nous allons présenter les enjeux qui ont été identifiés par ces acteurs-témoins, les stratégies d'action mises en avant et le regard qu'ils portent sur le bilan de leurs actions.

Si les témoignages recueillis dans le cadre du projet InterReconnaissance permettent de documenter partiellement l'émergence de la militance jeunesse LGBTQ, ils demeurent insuffisants à plusieurs égards pour en tracer un portrait exhaustif. Ainsi, le processus de sélection des participant.e.s à ce projet favorisait les activistes ayant exercé un leadership sur une période assez longue et acquis une notoriété suffisamment durable pour que leur nom soit proposé par leurs pairs lors des consultations préalables à la réalisation des entrevues. Ce processus est aussi biaisé en faveur d'une reconnaissance de la part de pairs issus d'organisations LGBTQ montréalaises. Les discours recueillis ne reflètent donc pas la diversité des expériences de militantisme autour des enjeux touchant les J-LGBTQ ni la variété des points d'insertion et des trajectoires des militant.e.s ayant débuté leur parcours d'activisme au cours des années 1990 ou 2000. Par exemple, plusieurs militant.e.s LGBTQ de la génération 2000 se sont investis dans des regroupements féministes, queers ou de la diversité ethnoculturelle, plutôt qu'autour des enjeux jeunesse. Par ailleurs, le projet InterReconnaissance souhaitait saisir les enjeux et les temps forts des luttes sociales du mouvement communautaire à partir de la mémoire qu'en ont des personnes dont l'implication a marqué leur secteur d'activisme sur une longue durée (Saillant et Lamoureux, 2018). Cet article met en valeur la parole d'acteurs-témoins du secteur LGBTQ, sans prétendre réaliser une analyse sociohistorique de l'émergence de la militance jeunesse LGBTQ.

\section{ANALYSE DES ENTREVUES}

\subsection{Motivations sous-jacentes à l'implication dans le mouvement LGBTQ}

À partir des discours des militants interviewés, on peut identifier trois catégories d'enjeux qui ont motivé leurs actions dans le mouvement LGBTQ : des expériences de discrimination vécues par les J-LGBTQ, la quasi-inexistence de services communautaires à leur intention et les interventions émanant des organismes publics considérées comme inadéquates pour répondre aux besoins des personnes LGBTQ.

\footnotetext{
${ }^{2}$ D'où notre emploi du masculin pour désigner ces interviewés. Un entretien amorcé avec une militante féministe et lesbienne n'a pu être complété concernant son implication comme activiste queer.
} 
À la suite de l'affirmation de leur orientation sexuelle, les militants rapportent avoir été rapidement aux prises avec plusieurs expériences de discrimination dans leur vie personnelle. Plusieurs relatent le malaise de leur famille et de leur entourage vis-à-vis de leur homosexualité, ce qui rend difficile l'expression de leur sexualité et engendre une crainte d'être rejeté. Érik Bisson évoque sa première relation amoureuse qui est demeurée secrète pendant trois mois : "Donc on vivait vraiment la vie clandestine. Et puis à un moment donné, on s'est dit : c'est difficile, on ne peut pas continuer comme ça. " Avant la reconnaissance des couples de même sexe et de leurs familles par les changements législatifs adoptés entre 1998 et 2005 (Tremblay, 2015), la vie homosexuelle impliquait aux yeux de plusieurs jeunes « de faire une croix sur le mariage, avoir des enfants, tout ça » (Erik Bisson), ce qui risquait en outre de décevoir les attentes de leurs parents. Ceux qui vivent éloignés des grands centres urbains, par exemple dans les régions de l'Abitibi-Témiscamingue, de Lanaudière ou du Bas-SaintLaurent, décrivent un isolement lié au manque d'information sur l'homo- ou la bisexualité et d'espaces de socialisation tels que des maisons de jeunes, des organisations communautaires où ils pourraient rencontrer des personnes qui partagent les mêmes préoccupations. Selon un témoignage, cet isolement est ressenti encore plus fortement chez les jeunes qui s'identifient comme trans, non binaires ou queers en dehors de Montréal. Les participants rapportent également des expériences de discrimination et de violence physique et verbale en raison de leur orientation sexuelle et de leur expression de genre, dont ils ont été victimes ou témoins dans diverses sphères de leur vie quotidienne. Par exemple, Bruno Laprade raconte avoir reçu des injures dans les rues de Montréal en raison de ses coupes de cheveux. Érik Bisson rapporte une expérience de discrimination dans son milieu professionnel au début des années 2000, alors qu'on lui refuse un congé quand son copain est malade.

On me dit: "Ah ben lui, ça compte pas. Tu ne peux pas avoir des journées de congé pour l'accompagner, lui, à l'hôpital, puisqu'il n'est pas considéré comme ton conjoint. Parce que ce n'est pas une fille. » Et donc moi, je me suis tourné de bord, j'ai appelé les ressources humaines, et puis j'ai dit qu'ils viennent me gérer ça, parce que moi, je vais sortir ça publiquement.

L'une des problématiques soulevées par les jeunes militants dès la fin des années 1980 porte sur la question de l'intimidation sur la base de l'orientation sexuelle vécue par les jeunes gais et lesbiennes dans les écoles, une question encore mal cernée à l'époque. En 1989, un sondage auprès de jeunes qui participaient au groupe de discussion du Comité d'intervention sociale de Jeunesse Lambda (le premier groupe d'entraide francophone pour J-LGBTQ mis sur pied à Montréal en 1987) révélait que le contexte scolaire du secondaire constituait l'un des environnements les plus difficiles à affronter dans le parcours de vie des J-LGBTQ. Jeunesse Lambda prend alors l'initiative de faire la tournée des écoles pour parler de l'organisme nouvellement créé. Cette initiative se trouve justifiée dès la première école visitée, car comme le rapporte Michel Mayrand, à l'arrivée des représentants du groupe, on leur annonce qu'un jeune élève a fait une tentative de suicide à cause des comportements homophobes dont il a été la cible.

On arrive à [École secondaire], puis là, le prof [...] nous dit : « Ah! Vous tombez bien ! On a eu un petit gars qui a fait une tentative de suicide à cause de l'homophobie à l'école. On a dû faire son coming out auprès de ses parents. On n'avait pas le choix. Ça n'a pas bien été. II est là, là-bas, on lui a dit ce que vous venez faire. » Puis là, on est là, la grosse goutte de sueur : "Bon! Ça commence bien. »

Un autre enjeu constaté par les participants au début de leur engagement est la quasi-inexistence des services, communautaires ou autres, à l'intention des J-LGBTQ. Michel Mayrand rapporte que dans les années 1980, à son arrivée à Montréal, le Lambda Youth, affilié à l'Université McGill, est le seul groupe 
actif pour J-LGBTQ, mais il ne tient des rencontres en français que de façon occasionnelle. Hugo Valiquette fait remarquer qu'en 1999, il n'y a aucune ressource pour les J-LGBTQ à Terrebonne dans la région de Lanaudière. À Montréal, dans les espaces existants pour la communauté LGBTQ et le Village gai, certains jeunes ne se sentent pas toujours les bienvenus. Bruno Laprade rappelle que les organismes pour les jeunes étaient peu présents à Montréal, au début de son implication entre 2000 et 2005. II relate également l'occurrence fréquente de sollicitations sexuelles et d'insultes à l'endroit des jeunes dont l'apparence déroge aux normes de genre, et relève l'absence à l'époque de lieux d'accueil sur d'autres bases que le consumérisme ou la sexualité.

En fait, les jeunes se plaignaient souvent aussi que le Village est un espace hyper sexualisé... qui apporte du positif, hein! Le but n'est pas simplement de faire du bad trash du Village, là ! Mais de reconnaître que, d'un côté, c'est un espace qui peut être d'appartenance, mais qui peut venir aussi avec des difficultés. Un côté commercial qui fait peur [...]. (Bruno Laprade)

En troisième lieu, les participants se montrent critiques vis-à-vis de l'inaction du gouvernement ou de la manière dont celui-ci intervient pour régler certains problèmes, qui ne concernent pas exclusivement les jeunes. En termes d'enjeux, les lois occupent peu de place dans le discours des participants. Ces derniers rapportent plutôt que l'enjeu est de changer les règles et les politiques au sein des institutions publiques: par exemple, assouplir les procédures du Directeur de l'état civil pour effectuer un changement de prénom et obtenir des papiers d'identité correspondant au genre d'identification pour les personnes trans, faire adopter ces changements par les établissements scolaires, en particulier les cégeps et les universités, et par les institutions publiques en général, adapter les installations physiques (par ex., toilettes non genrées). Trois participants nomment les réglementations pour les dons de sang comme des restrictions discriminatoires, car ces règles s'appliquent exclusivement aux hommes ayant des relations sexuelles avec d'autres hommes et entretiennent ainsi une représentation négative au sujet des hommes gais.

Avant, ça posait la question qui était totalement absurde, c'est : " Avez-vous eu une relation sexuelle avec un autre homme depuis 1977 ? " Puis on était en 2012, 2013. Ça présumait que si tu avais eu une relation sexuelle avec un [homme], aujourd'hui, tu es potentiellement séropositif, et donc on ne peut pas prendre ton sang. Un, je le saurais si je suis séropositif. Ce n'est pas parce que j'ai des relations sexuelles avec un autre homme qu'elles ne sont pas sécuritaires. Maintenant c'est rendu à : "Avezvous eu une relation sexuelle avec un autre homme dans les cinq dernières années ? "C'est moins pire, mais c'est encore aussi absurde. [...] En fait, ils ne demandent pas ça aux hétérosexuels. (Robert Pilon)

Un autre participant dénonce l'inefficacité des mesures de prévention du VIH et l'obligation pour les personnes vivant avec le VIH de dévoiler leur statut sérologique sous peine d'être criminalisées : "Maintenant que les personnes séropos ne meurent plus, elles sont mises en prison par rapport au non-dévoilement de leur statut sérologique. » (Bruno Laprade)

\subsection{Les stratégies d'action}

Trois types de stratégies d'action sont privilégiés pour répondre à ces problèmes : la mise en place de services communautaires par et pour les J-LGBTQ et les efforts pour les regrouper ; des interventions de sensibilisation dans les écoles; des manifestations et actions directes de type Zap visant à sensibiliser le milieu LGBTQ et le grand public à certains enjeux. 


\subsubsection{La création d'organismes par et pour les jeunes}

Les interviewés relatent la création d'organismes communautaires qu'ils ont contribué à mettre sur pied dans diverses régions du Québec ou leur implication dans des organismes déjà existants. Ainsi, Michel Mayrand raconte I'historique de Jeunesse Lambda, le plus ancien d'entre eux, dont l'ancêtre Lambda Youth, fondé dans l'ouest de Montréal, date de 1976. Dans les années 1980, Lambda Youth devient Jeunesse Lambda Youth, un organisme bilingue avec une section francophone et une rencontre mensuelle en français, puis celle-ci s'autonomise pour devenir Jeunesse Lambda en 1987. Au sein de ce dernier, un comité est créé pour faire des interventions de démystification de l'homosexualité dans des écoles. C'est le Comité d'intervention sociale qui, selon Mayrand, voit le jour en 1989 et deviendra un organisme autonome connu sous le nom de GRIS, appelé à prendre de l'expansion de façon exponentielle dans les décennies suivantes. Marc-Olivier Ouellet et Bruno Laprade ont milité au sein de Projet 10, un organisme fondé en 1990 qui visait à promouvoir le bien-être des jeunes ayant entre 14 et 25 ans, entre autres en leur offrant des espaces sécuritaires informels (drop in). Un autre organisme, Jeunesse Idem, naît en 1995, d'abord sous la forme d'un programme mis en place par le Bureau régional d'Action Sida (BRAS) afin de développer des activités d'éducation préventive VIH/sida et de promotion de la santé spécifiques aux besoins et intérêts des hommes de l'Outaouais qui ont des relations sexuelles avec d'autres hommes. II se transforme par la suite en organisme venant en aide aux jeunes allosexuel.le.s de 14-25 ans qui s'identifient comme LGBTQ ou se questionnent sur leur orientation sexuelle ou leur identité de genre. C'est au sein de cet organisme que débute la trajectoire militante d'Érik Bisson : « J'ai commencé en 96 comme participant à Jeunesse IDEM. Ça a été vraiment le début d'un bien-être personnel et d'une implication sociale collective. » Bénévole pendant sept ans, il occupe ensuite le poste d'intervenant. En 1999, Hugo Valiquette crée la Fondation Néo pour pallier l'absence de services pour les jeunes dans la région de Lanaudière. Après son coming out, il s'était rendu compte qu'il n'y avait pas d'organisme desservant cette population. Bien qu'il fût lui-même bien accepté par sa famille et son entourage, il savait que ce n'était pas le cas pour d'autres jeunes de la région. Le Néo innove en mettant sur pied des services et un milieu de vie pour les jeunes, mais aussi une ligne d'écoute par et pour les jeunes et un volet d'intervention sur le Web.

Au Néo, on avait la ligne d'écoute, puis pour nous, c'était important que ce soit un [organisme] par et pour les jeunes. On avait développé aussi tout un volet d'intervention par le Web. Puis c'était quand même pionnier au début des années 2000, les sites Web, c'était assez limité. C'était une vitrine. Nous, on avait déjà un forum, on avait un chat directement sur la page Web. On se gardait vraiment à la fine pointe des sites Web. On avait du Flash. On investissait beaucoup au niveau du Web, on se disait : les jeunes, on le sait qu'ils ont ce besoin-là, d'anonymat, de confidentialité. [...] Il y avait tout le volet groupes de discussion, le volet drop in, groupes de soutien aux parents... (Hugo Valiquette)

En 2002, Marc-Olivier Ouellet mise également sur l'Internet en concevant un portail en ligne, AlterHéros, afin de démystifier la diversité des orientations sexuelles et des identités de genre auprès des jeunes. Olivier Poulin cofonde le groupe Arco Iris au Cégep de Sainte-Foy en 2000-2001 avec son ami Olivier Vallerand et le soutien de l'intervenant social Antoine Coulombe ${ }^{3}$, puis milite au sein du Groupe gai de I'Université Laval - créé en 1978, ce qui en fait le plus ancien groupe LGBTQ universitaire du Québec. Étonné par l'absence d'un tel organisme au sein de l'Université du Québec à Montréal, B. participe à la

${ }^{3}$ Sur la création de l'organisme Arco-Iris ainsi que le développement de l'intervention visant l'empowerment des jeunes LGB, voir Coulombe, 2008. 
création de La Réclame, un groupe de socialisation et de défense des droits des personnes LGBTQIA+ qui veut aussi accueillir des étudiant.e.s en provenance des régions. Dans les années 2000, les groupes collégiaux et universitaires se multiplient, mais faute de ressources et de soutien institutionnel, leur existence est souvent éphémère. L'historique de ces organismes reste à faire.

\subsubsection{La création de regroupements}

C'est dans les années 2000 que le militantisme jeunesse acquiert une visibilité au sein du mouvement LGBTQ avec la formation d'alliances et la mise en place d'organisations qui fédèrent les organismes par et pour les J-LGBTQ et offrent des lieux de concertation pour les militant.e.s. C'est le cas du Regroupement d'entraide pour la jeunesse allosexuelle du Québec/Quebec Alliance for Queer Youth (REJAQ/QAQY), créé en 2001 (Laprade, 2013), qui se veut une fédération à l'échelle provinciale, et de la Coalition jeunesse de lutte contre l'homophobie à Montréal (CJLHM, actuellement la Coalition montréalaise des groupes jeunesse LGBT) qui vise une concertation entre les organismes LGBTQ offrant des services aux jeunes dans cette ville. Ces deux regroupements se veulent inclusifs quant à la diversité des genres et des sexualités. Ainsi, le REJAQ, dès 2002, propose le terme " allosexuel » comme traduction de queer. Ils se font entendre dans divers espaces politiques, notamment ceux qui se préoccupent des jeunes. La CJLHM est active au sein du Forum jeunesse de l'île de Montréal, où un siège est réservé dans le conseil d'administration pour la représentation des jeunes allosexuel.le.s et qui deviendra un partenaire majeur pour la réalisation de ses projets. De son côté, le REJAQ devient membre de la Table des regroupements provinciaux d'organismes communautaires et bénévoles, présente des mémoires lors de consultations publiques sur la stratégie d'action jeunesse du gouvernement du Québec pour la deuxième moitié des années 2000 et organise à partir de 2006 le Gala des allostars qui valorise et promeut l'engagement de la jeunesse allosexuelle du Québec (pour plus d'informations, voir Laprade, 2013). Cette stratégie d'autonomisation et d'occupation des espaces politiques destinés aux jeunes leur permet d'acquérir progressivement une voix politique propre plutôt que d'être dépendants des organisations reconnues comme porte-parole officiels du mouvement LGBTQ.

Les organismes communautaires et les regroupements J-LGBTQ sont alors confrontés de manière continue à un défi majeur, soit le sous-financement chronique qui fragilise le réseau de services par et pour les jeunes. Sans ressources financières, il est non seulement difficile de maintenir le personnel et l'offre de services, mais aussi, pour les organismes, de tenir un lieu physique permanent. Selon les témoignages, les militants se butent à un déni de la réalité et des besoins des J-LGBTQ par les élu.e.s et par les administrations municipales et régionales, ce qui complique leur accès aux ressources matérielles (soutien financier, local). Ils doivent sans cesse démontrer la pertinence du travail de leurs organisations et faire valoir leur crédibilité. Bruno Laprade souligne le manque de considération fondé sur l'âge, affirmant que les jeunes leaders voient leur crédibilité remise en question de plusieurs manières du fait de préjugés âgistes et d'aprioris selon lesquels le problème de l'homophobie cesserait ou aurait cessé dans un contexte d'égalité juridique. Pour Hugo Valiquette et Érik Bisson, le manque de financement a été l'une des premières causes de la disparition du REJAQ, tenu à bout de bras par des bénévoles jusqu'en 2009, qui était pourtant parvenu à rassembler des organismes issus de plusieurs régions du Québec et des associations étudiantes LGBTQ collégiales et universitaires. Outre la participation aux activités des uns et des autres, ce regroupement favorisait les échanges de bonnes pratiques et la concertation : " Comme on travaillait beaucoup ensemble au niveau du REJAQ, ça nous donnait des idées pour nos organismes. Le GRIS-Québec avait aussi voulu mettre en place un milieu 
de vie [pour les jeunes], alors on leur avait fourni notre demande de financement, répondu à des questions. J'étais allé les rencontrer, leur présenter plein d'affaires. [...] L'alliance, c'était le REJAQ. » (Hugo Valiquette) Un autre exemple illustre l'instabilité générée par l'insuffisance de ressources : la même année, Jeunesse Lambda et Projet 10 ont été forcés de déménager de leurs locaux, sans avoir de solution de remplacement satisfaisante.

À Jeunesse Lambda, l'année où on s'est fait mettre à la porte aussi du restaurant [que fréquentaient régulièrement des membres de Jeunesse Lambda après le groupe de discussion du vendredi], le building où on était menaçait de s'écrouler. On a été trois mois à devoir faire nos activités ailleurs. En même temps, Projet 10 cherchait à déménager. Donc on sentait qu'il y avait une fragilité des groupes jeunesse LGBT qui faisaient un travail qui, pour nous, était considéré comme nécessaire. (Bruno Laprade)

Pour répondre à ces difficultés, les militants montréalais vont notamment poursuivre le projet de créer un milieu de vie, un espace où les organismes par et pour les J-LGBTQ pourront offrir leurs services tout en partageant les locaux et où diverses activités pourront avoir lieu : "Donc on voulait ouvrir un espace pour les jeunes où les gens n'auraient pas une obligation de consommer, ou pourraient se rencontrer sur une autre base que justement, le flirt. » (Bruno Laprade) La CJMLH regroupe un nombre croissant d'organismes jeunesse ou intervenant relativement à des questions qui touchent les jeunes dans la région montréalaise et, contrairement au REJAQ, elle a pu finalement bénéficier d'un soutien financier qui a assuré sa survie. Après une quête d'appuis longue et frustrante par moments, ainsi que le rapporte Laprade, le projet voit enfin le jour avec la mise sur pied de l'Astérisk en 2013, qui assure un espace stable et sécuritaire aux organismes jeunesse et une visibilité aux jeunes dans le Village gai : “On avait fait plein de démarchage. [...] On se heurtait beaucoup à des portes closes jusqu'à tant qu'on arrive avec le Forum jeunesse qui a vraiment été [d']une plus grande écoute puis qui a décidé de financer un projet pour la diversité sexuelle en 2009-2010. 》(Bruno Laprade)

\subsubsection{Les interventions en milieu scolaire}

Les interventions en milieu scolaire constituent une autre stratégie expérimentée au milieu des années 1990 et déployée à plus vaste échelle dans la décennie suivante. II s'agit de démystifier les préconceptions sur l'homosexualité par l'approche du témoignage. Cette initiative du Comité d'intervention sociale (CIS) de Jeunesse Lambda fait l'objet de commentaires positifs, ce qui lui donnera un bon élan. Les interventions se multipliant, le CIS grossit et commence à avoir un budget plus important que Jeunesse Lambda. En 1994, ce qui se présentait comme un projet mené par un comité subalterne se transforme en un organisme autonome, le Groupe de recherche et d'intervention sociale (GRIS-Montréal), dont le modèle d'intervention sera repris dans plusieurs régions du Québec sous l'appellation de GRIS de même que par des organismes jeunesse offrant divers types de services et d'intervention. Robert Pilon décrit la formule : un duo d'intervenant.es bénévoles se rend dans les classes pour discuter de la pluralité des vécus en lien avec l'orientation sexuelle auprès des jeunes, et aborder ouvertement des questions qui sont au cœur de leur inconfort ou de leur incompréhension. L'amélioration constante de la formule (recrutement et formation de bénévoles, confection d'outils publicitaires, notamment avec des vedettes alliées bien connues du public, etc.) contribuera à son succès :

L'année avant que j'arrive au GRIS, ils faisaient une trentaine de classes par année. Quand je suis arrivé, on en a peut-être fait le double, 70, puis dès l'année de cette carte-là [publicité avec des 
vedettes qui se sont alliées à la cause], on est montés au-dessus de 100, puis c'est monté : 200,400 , 500,800 . Là, on est rendus en moyenne à 1000 classes par année. Ça n'a jamais monté d'un coup sec, mais d'année en année, ceux qui nous appellent continuent de nous appeler. (Robert Pilon)

Mentionnons également le Coffret d'intervention en milieu scolaire, une initiative à laquelle a participé AlterHéros, qui consistait à distribuer dans les écoles des coffrets comprenant des vidéos, guides, dépliants, ressources et affiches de diverses organisations pour jeunes.

\subsubsection{Les actions directes}

Les manifestations et les actions directes constituent d'autres stratégies d'action qui sont privilégiées, notamment par des activistes queers et non binaires qui collaborent avec des militant.e.s anglophones et de différents milieux. Des organisations comme PolitiQ - collectif activiste Queers solidaires de Montréal (2009-2014) se donnent un mandat de sensibilisation politique et d'éducation populaire, privilégient des actions directes et des manifestations qui intègrent la performance artistique, avec plusieurs mises en scène. Leurs actions contestent le système économique et social, et non seulement les discriminations envers les personnes LGBTQ. Par exemple, des manifestations visaient à dénoncer la brutalité policière et les arrestations arbitraires dans le contexte de la grève étudiante contre l'augmentation des frais de scolarité en 2012, ou encore les décisions du gouvernement conservateur à Ottawa et les politiques néolibérales, comme le raconte Bruno Laprade :

[Lors d'une parodie de remise de prix appelée le Gala des osties de racistes] on avait écrit un speech, justement, pour parler de l'œuvre de Stephen Harper en matière de racisme, mais aussi d'homophobie. Sa manière aussi de couper les vivres aux groupes féministes. Donc faire un peu le lien entre l'ensemble de son œuvre pour dire à quel point il était fier d'accepter ce prix qui remettait en cause, en fait, les questions des droits de plusieurs Canadiens.

Les mobilisations soutiennent également les revendications en faveur des personnes trans, notamment celles concernant le changement de prénom et de la mention de sexe.

\section{LE BILAN DES ACTIONS MENÉES}

Le bilan de leurs actions dressé par les militants interviewés est globalement positif, quoiqu'il s'avère quasi impossible d'isoler les effets attribués à leur propre engagement du regard qu'ils jettent sur les transformations sociales de la société face à la diversité sexuelle et de genre. Les activités menées ont d'abord eu un effet positif sur les participants eux-mêmes, avec une amélioration de leur estime de soi à la suite de leur implication militante, qui s'est poursuivie pendant plusieurs années. Plusieurs participants expriment le sentiment d'avoir contribué à transformer pour le mieux les conditions de vie des J-LGBTQ, soit directement par l'action de leur organisme, soit par la participation à un ensemble d'actions qui ont engendré un changement globalement positif. Ainsi, Marc-Olivier Ouellet d'AlterHéros exprime sa fierté d'avoir réussi à joindre via Internet plusieurs milliers de personnes à travers la francophonie, dont un très grand nombre de jeunes. Robert Pilon, du GRIS-Montréal, rappelle la notoriété croissante de l'organisme qui est de plus en plus sollicité par les établissements scolaires. L'organisme a aussi mené une campagne s'adressant aux parents, à l'aide d'une carte postale où figuraient des comédiens connus et la phrase : « Nos enfants ne seront pas homophobes. » Au-delà du succès quantitatif, il remarque que les commentaires qui paraissent dans les questionnaires d'évaluation des interventions démontrent l'impact positif des interventions du GRIS sur la vie des jeunes, tout en soulignant le travail qui reste à faire pour démystifier la diversité des réalités LGBTQ. II 
en donne pour exemple les propos d'un jeune qui relevait qu'à la suite du passage du GRIS dans son école, ses camarades s'étaient montrés plus ouverts à son égard :

Il avait dit: «Quand vous êtes passés dans mon école, c'est comme si vous aviez passé l'aspirateur de l'homophobie. » [...] Puis là, il dit : « Du monde qui me parlait pas avant ou qui me criait des noms dans le corridor venaient me saluer, puis dire : "Hein! Salut! T'es gai. C'est cool. On en a parlé l'autre fois." » Lui, il n'en revenait pas que du jour au lendemain, ça avait fait changer la façon d'être perçu par les autres. Donc tu te dis : «II y a quelque chose qui change. » (Robert Pilon)

II se dit gratifié également par les témoignages de jeunes qui, des années plus tard, le reconnaissent comme intervenant du GRIS et lui expriment combien ils ont apprécié son passage à leur école. Olivier Poulin voit un indice positif des changements survenus dans le fait que des élèves non LGBTQ s'impliquent dans la lutte contre l'homophobie et la transphobie, alors qu'auparavant, ils évitaient toute association avec cette cause de peur d'être eux-mêmes étiquetés comme tels : “C'est une chose formidable, une révolution qui donne confiance dans l'avenir. » Michel Mayrand fournit un autre exemple qui donne la mesure du chemin parcouru, celui d'un « beau reportage » sur une «fille androgyne » à son bal des finissants de l'école secondaire : “Elle était chanceuse, c'était une école ouverte, elle n'a pas été ostracisée. Mais [dans] plein d'autres endroits, ça aurait pu être pire. » Ce dernier bémol est formulé par certains participants : les écoles seraient inégalement rejointes par les interventions de sensibilisation à la diversité sexuelle et de genre, en raison du manque de ressources des organismes LGBT, de l'étendue des territoires à couvrir dans certaines régions et du degré d'engagement variable des autorités scolaires alors qu'il y a parfois un déni de la présence de J-LGBT dans les écoles.

En matière de collaboration externe, les participants notent que les organisations jeunesse ont su s'insérer dans le réseau communautaire LGBT, en créant des alliances avec les organisations déjà présentes, mais aussi avec d'autres secteurs communautaires et avec le gouvernement. Selon la plupart, les organismes jeunesse trouvent maintenant une écoute de la part des élu.e.s, des institutions scolaires et municipales. Des exemples en sont rapportés, comme la visite d'une ministre à l'occasion d'un défilé de la Fierté et sa disponibilité pour échanger avec les jeunes. La présence de personnalités publiques, principalement du monde politique, est vue comme un signe de soutien à l'action des organismes jeunesse. Une autre victoire est d'avoir été capable d'insérer les besoins des J-LGBTQ et leurs revendications dans les préoccupations politiques à divers niveaux (par exemple, dans certains rapports municipaux et dans les documents relatifs aux stratégies provinciales d'action jeunesse) et par la suite d'avoir obtenu un appui financier pour leur organisme ou pour un projet comme l'Astérisk. Cependant, l'appréciation du chemin qu'il a fallu parcourir pour obtenir des appuis varie d'un participant à l'autre. Alors qu'Hugo Valiquette évoque le soutien immédiat des élus locaux et des institutions publiques à la création du Néo dans la région de Lanaudière, d'autres, comme Bruno Laprade, estiment que ce sont les nombreuses mobilisations de terrain qui ont fini par vaincre les résistances et conscientiser les milieux institutionnels dans les domaines éducatif, de la santé et municipal. Il en donne comme exemple une tournée organisée par PolitiQ, la CJLHM et l'Association pour une solidarité syndicale étudiante (ASSÉ) en 2011 auprès des étudiant.e.s dans les cégeps et les universités pour les sensibiliser aux enjeux LGBTQ. II reste que les institutions d'enseignement supérieur demeurent réticentes à effectuer des changements concrets pour faciliter l'usage du prénom choisi et réaliser des aménagements pour les personnes trans dans les toilettes et vestiaires. Laprade rappelle également que les positions plus radicales de groupes s'inscrivant dans les perspectives queers comme PolitiQ étaient parfois mal perçues, jugées infondées ou mal comprises. 
Les participants formulent aussi un jugement nuancé sur la situation des personnes LGBTQ, tous groupes d'âge confondus, et les enjeux actuels. Plusieurs soulignent les changements positifs dans l'opinion publique, soit une meilleure acceptation de la diversité des orientations sexuelles et des identités de genre ainsi qu'une bonne réception des actions de mobilisation. Certains émettent toutefois des doutes sur la profondeur de tels changements. Si la désapprobation de l'homophobie fait l'objet d'un certain consensus social, les préjugés concernant les personnes LGBTQ persisteraient même s'ils s'expriment moins ouvertement qu'avant. Selon Érik Bisson, les gens ne reconnaissent pas les manifestations d'hétérosexisme, «soit par exemple de tenir pour acquis que les personnes sont hétérosexuelles ou que les couples fonctionnent sur le modèle hétérosexuel, ou les gens n'acceptent pas que les couples gais peuvent élever des enfants ". La couverture médiatique des activités autour de la Fierté LGBTQ est généralement qualifiée de positive, car elle donne de la visibilité aux actions des organismes LGBTQ et à l'engagement de personnalités publiques à leurs côtés. Par exemple, Olivier Poulin rapporte l'important impact médiatique (journaux, radio et réseaux sociaux) de l'appui du maire Régis Labeaume de la ville de Québec lorsqu'il a accepté de hisser le drapeau arc-en-ciel à l'hôtel de ville lors des Jeux olympiques de Sotchi en Russie. Cependant, l'attention médiatique n'est pas toujours bénéfique. Hugo Valiquette donne l'exemple d'un incident homophobe (drapeau de la fierté brûlé) qu'il a dénoncé par un communiqué de presse ; par la suite, il s'est vu reprocher de donner une mauvaise image de la ville en raison de la forte attention médiatique accordée à l'évènement. D'autres participants portent un regard critique sur l'instrumentalisation médiatique de certains enjeux. Par exemple, l'intérêt récent à l'égard des questions trans de la part de journalistes ou d'étudiant.e.s qui en font le sujet d'un travail académique relèverait souvent d'une curiosité malsaine, du sensationnalisme et du voyeurisme, sans retour positif pour les militant.e.s qui collaborent avec eux. Au moment des entrevues, les revendications trans n'avaient toujours pas obtenu satisfaction sur le plan législatif ${ }^{4}$ et plusieurs participants en relèvent l'importance. Bruno Laprade insiste sur le rôle de PolitiQ dans la sensibilisation des autres organismes LGBTQ par rapport à ces revendications. D'autres questions, émergentes ou n'ayant toujours pas trouvé de solution satisfaisante, sont mentionnées parmi les enjeux actuels : la reconnaissance de l'homoparentalité dans les diverses sphères sociales dont l'école, l'accès à la parentalité pour les hommes gais à l'aide de la gestation pour autrui, les restrictions au don de sang, la criminalisation de la non-divulgation de son statut sérologique, les droits des enfants trans et des personnes intersexes. Enfin, les deux militants queers amènent des perspectives intersectionnelles en soulignant la nécessité d'appuyer les luttes contre le racisme et le sexisme ainsi que les luttes en faveur de groupes plus marginalisés comme les travailleur.euse.s du sexe et les immigrant.e.s LGBTQ.

\section{Discussion}

Cette recherche a permis d'esquisser quelques contours de l'émergence d'une militance autour des enjeux touchant les J-LGBTQ, en examinant les situations et les expériences indiquées comme ayant motivé l'engagement dans le mouvement LGBTQ, les stratégies d'action ainsi que le bilan qu'en font les militants interviewés.

Les participants évoquent des expériences de stigmatisation, vécues ou appréhendées, dans leur milieu familial, à l'école et dans leur entourage à la suite de la divulgation de leur orientation sexuelle ou de

\footnotetext{
${ }^{4}$ Depuis, des changements législatifs sont intervenus pour faciliter le processus de changement de prénom et de la mention de sexe dans l'état civil, et l'identité et l'expression de genre ont été ajoutées comme motifs interdits de discrimination dans la Charte des droits et libertés de la personne du Québec ainsi que dans la Loi canadienne sur les droits de la personne.
} 
leur identité de genre ; des discriminations en milieu professionnel ; leur isolement dans des régions hors des grands centres urbains, et même à Montréal où, avant les années 2000, les jeunes disposaient de peu de ressources ; et finalement, la violence physique et verbale dans des lieux publics sur la base de l'orientation sexuelle ou de l'expression de genre. Ces données vont dans le même sens que celles d'autres recherches menées sur les J-LGBTQ au Québec et ailleurs, parmi lesquelles plusieurs ont documenté les impacts de la stigmatisation et du rejet social sur les J-LGBTQ (voir entre autres Chamberland et Dorais, 2013 ; Blais, Gervais et Hébert, 2014 ; Dorais, 2014a et 2014b ; Cénat et al., 2015 ; Veale et al., 2015 ; Lépine et al., 2017). Il en ressort des éléments de continuité et de discontinuité par rapport aux militant.e.s des générations antérieures (Chamberland et al., 2018). Les jeunes rapportent des expériences difficiles similaires à celles des générations précédentes, telles que l'ostracisme à l'école et la crainte du rejet qui rend malaisée la divulgation aux parents et à l'entourage. Cependant, leurs propos font plus souvent référence à des incidents spécifiques ou à des situations temporaires plutôt qu'à une condamnation généralisée de l'homosexualité comme c'est le cas dans les témoignages des militant.e.s plus âgés. II reste que la persistance des expériences de discrimination et d'intimidation dans un contexte où des dispositifs législatifs et juridiques ont été mis en place soulève la question de la modification en profondeur des attitudes sociétales, qui va au-delà de l'interdit formel de discriminer en raison de l'orientation sexuelle.

La question du VIH est un défi qui perdure également, mais contrairement aux témoins de la génération qui a connu le choc de la pandémie dans les années 1980-1990, elle n'est abordée que par une minorité des militants interviewés, et ce, en référence à la criminalisation de la non-divulgation de la séropositivité et aux nouveaux enjeux de prévention tels que les autotests. Autrement dit, il s'agit surtout de diffuser l'information en santé préventive. Les ressources déployées pour la prévention du VIH ont aussi parfois constitué un point de départ stratégique pour la mise en place d'organismes intervenant par rapport à la santé globale des jeunes hommes, devenus par la suite plus inclusifs de la diversité sexuelle et de genre, comme c'est le cas de Jeunesse Idem, mentionné précédemment, ou d'un organisme comme Mains Bas-Saint-Laurent (voir le site Web www.mainsbsl.qc.ca). Par ailleurs, le statut des personnes trans prend une place beaucoup plus grande qu'auparavant dans le discours politique de plusieurs participants étant donné que ni l'égalité juridique ni la reconnaissance sociale ne sont atteintes pour les personnes trans, ce qui constitue une particularité des générations militantes des années 2000 ainsi que le révèlent également les dénominations de certains organismes ou regroupements comme « allosexuels » (sur l'émergence du mouvement trans francophone à Montréal, voir Enriquez, 2013, 2014 et Pabion, 2016).

Les stratégies d'action mises en place par les militants renvoient principalement à la création d'organisations communautaires : des organismes qui ciblent spécifiquement les 14 à 25 ans et leur proposent diverses activités (entraide, discussion, etc.) dans une perspective d'autonomisation, des associations étudiantes en milieu collégial et universitaire, des organismes d'intervention dans le milieu scolaire comme les GRIS qui vont faire leur apparition dans plusieurs régions du Québec au cours des décennies suivantes. La création d'organisations communautaires pour pallier le manque de services orientés vers les J-LGBTQ correspond à une stratégie déjà bien éprouvée dans les phases antérieures du mouvement LGBTQ, mais leur originalité renvoie au fait qu'ils sont mis en place par et pour les jeunes et couvrent l'ensemble du spectre de la diversité sexuelle et de genre, ce qui constitue une nouvelle orientation dans la structuration de ce mouvement. Cette stratégie d'action s'observe également à Toronto où les jeunes LGBTQ, par le biais de l'organisation SOY, ont mis en place des initiatives et des ressources par et pour les jeunes visant à améliorer leurs conditions et leur qualité de 
vie, en leur offrant du soutien, en répondant à leurs besoins matériels (logement), en répondant aux besoins des plus marginalisés et en offrant des activités d'expression artistique (Grundy et Smith, 2005, p. 397-399).

On peut noter aussi que l'absence de ressources financières pour les organismes jeunesse québécois est un défi auquel est confronté le réseau des organisations communautaires LGBTQ depuis toujours. Toutefois, la spécificité dans leur cas est l'injonction qui leur est faite de démontrer leur crédibilité alors qu'existent d'autres organisations et porte-parole du mouvement LGBTQ mieux connus et ayant acquis une reconnaissance dans leurs rapports avec le gouvernement et les autorités publiques (Laprade, 2013). Des organisations fédératives émergent également dans la décennie 2000, telles que le REJAQ et la CJLHM, confirmant l'importance de l'établissement d'alliances internes et externes, comme cela a été noté pour l'obtention de gains législatifs touchant les couples de même sexe et leurs familles (Chamberland et al., 2018). Pour leur part, les organismes et regroupements jeunesse sont parvenus à sensibiliser différents acteurs clés au sein des organismes LGBT, des associations syndicales d'enseignant.e.s et des autorités scolaires et gouvernementales à propos des impacts de l'intimidation sur les J-LGBTQ. Leur cause fut également relayée par la Commission des droits de la personne et des droits de la jeunesse (2002) et par le Conseil permanent de la jeunesse (2007). Les mobilisations d'une pluralité d'acteurs dans le secteur éducatif ont débouché sur un éventail de stratégies et de pratiques d'intervention pour transformer le climat scolaire au Québec (Richard et al., 2015 ; Chamberland et Richard, 2017) et l'on oublie trop souvent le rôle des organismes jeunesse LGBTQ qui ont sonné l'alerte dès la décennie 1990 et suscité cet engagement de leur part.

Par ailleurs, des militants de cette génération renouent avec les mobilisations et les actions directes, utilisant des stratégies de dénonciation publique et de mises en scène performatives inspirées par le courant politique queer. Toutefois, le trop petit nombre de participants animés par ce courant ne permet pas de retracer ici la présence et l'influence de ce courant ni les interactions avec les autres composantes de la militance jeunesse. Sur l'émergence du mouvement queer francophone à Montréal, on pourra consulter Pabion, 2016 de même que les sites https://infopolitiq.wordpress.com et http://www.lespantheresroses.org.

Sans que cela représente un thème très développé dans les discours des participants, on peut noter l'usage de plateformes Internet par ces organisations, ce qui constitue une nouvelle étape dans les stratégies de soutien, d'intervention, de mobilisation et de collaboration sur les plans local et international du mouvement LGBT (voir Dumas et al., 2010 ; Lévy et al., 2011 ; Lévy et Dumas, 2012). Une autre particularité de l'activisme jeunesse LGBTQ est que la judiciarisation (recours aux tribunaux) et la juridification (appropriation du langage des droits) des luttes (Smith, 2011 ; Paternotte, 2012 ; Chamberland et al., 2018) semblent moins observées dans les pistes d'action mises en avant par les nouvelles générations concernant les droits des gais et lesbiennes, et même par rapport aux personnes trans qui demeurent pourtant privées d'une reconnaissance et d'une protection juridiques - ce qui contraste donc avec la prédominance d'un discours axé sur les droits et l'égalité juridique qui constituait la principale orientation stratégique de l'activisme de la génération précédente de militant.e.s. Dans l'ensemble, ce militantisme jeunesse présente donc des particularités qui lui confèrent une couleur spécifique par rapport au reste du mouvement.

Bien que peu de participants mettent en valeur leur rôle particulier dans les changements sociaux opérés, la plupart font un bilan positif de leur engagement et des actions menées, autant par rapport à leur vie personnelle que par rapport à celle des J-LGBTQ. Nous pouvons ajouter que plusieurs ont milité 
pendant une période relativement longue, qu'ils ont affirmé leur leadership au fil des ans et acquis une relative notoriété au sein de la militance jeunesse LGBTQ ainsi que dans le mouvement LGBTQ québécois. C'est d'ailleurs cette notoriété, confirmée par la réception de prix communautaires dans plusieurs cas, qui explique leur présence dans cette étude. La composition principalement masculine de notre échantillon nous amène à observer que ce sont surtout de jeunes gais, et non de jeunes lesbiennes, qui ont exercé un rôle de premier plan dans la structuration de la militance jeunesse LGBTQ, du moins jusqu'à ces dernières années. Ces militants incarnent une identité politique distinctive par rapport au reste du mouvement, axée sur des enjeux sociaux concernant spécifiquement les jeunes. Les années 2000 ont vu émerger d'autres militances portées par des jeunes activistes appartenant à la même cohorte générationnelle, mais centrées sur d'autres enjeux. Par exemple, Arc-en-ciel d'Afrique et Helem, deux groupes fondés en 2004 et ciblant respectivement les personnes LGBTQ afrocaribéennes et les personnes LGBTQ d'origine libanaise et arabophones, sont des organismes créés et fréquentés principalement par des jeunes et de jeunes adultes, mais ils ne se définissent pas en termes générationnels, ni dans la description de leur membership, ni dans celle des enjeux qui les touchent. De même, au début des années 2010, la plateforme en ligne LSTW (Lez spread the word) cible principalement les lesbiennes jeunes, mais sans en faire une caractéristique identitaire ni articuler un discours spécifique sur les jeunes.

Cette recherche présente d'importantes limites découlant notamment de l'orientation générale du projet, axé sur la mémoire des luttes pour les droits. L'analyse se base sur les propos des participants, lesquels comportent des lacunes et des imprécisions quant aux dates et aux contextes précis de leurs actions. Malgré la valeur des témoignages recueillis, il ne s'agit pas d'un véritable travail historiographique. De plus, le petit nombre d'entrevues ainsi que le processus de sélection des participants, basé sur leur notoriété auprès d'un comité consultatif composé de Montréalais.e.s, ont eu pour effet de négliger la participation aux luttes LGBTQ de militant.e.s non francophones, d'origine immigrante, non blancs et résidant hors de Montréal. Le militantisme de lesbiennes et de femmes bisexuelles impliquées dans des réseaux non mixtes (comme le Réseau des lesbiennes du Québec ou le magazine Sapho à Québec) ou dans d'autres sphères d'activisme (féministe, queer, etc.) est également passé sous silence.

\section{Conclusion}

Malgré son incomplétude, cet article a permis de retracer quelques caractéristiques de l'émergence de la militance autour des enjeux touchant les J-LGBTQ au Québec dans les années 1990 et 2000. II rappelle quelques jalons dans le long processus de construction d'un réseau de soutien par et pour les jeunes ainsi que la créativité des premières associations qui ont mis l'accent sur l'autonomisation des jeunes, développé un modèle original d'intervention sur le terrain scolaire basé sur l'approche du témoignage et misé sur les technologies du Web pour rejoindre et aider les J-LGBTQ tout en respectant leur besoin de confidentialité. De plus, les enjeux concernant les J-LGBTQ ont acquis une visibilité sociale et politique, comme en témoignent le rapport de la Commission des droits de la personne et des droits de la jeunesse qui a inspiré l'adoption d'une politique gouvernementale contre l'homophobie (Groupe de travail mixte sur l'homophobie, 2007) ainsi que les mesures contenues dans le Plan d'action gouvernemental de lutte contre l'homophobie et la transphobie, 2017-2022 (Ministère de la Justice, 2017) et la Stratégie d'action jeunesse 2016-2021 du Secrétariat à la jeunesse (2017). Bien que le réseau d'organismes communautaires mis sur pied par et pour les J-LGBTQ demeure confronté à des défis criants, il apparaît que l'impact de ces organismes a été déterminant pour une génération de J- 
LGBTQ qui ont pu accéder à des informations et à des ressources dans des moments de détresse. ॥ est souhaitable que des travaux futurs approfondissent l'histoire du militantisme jeunesse au Québec tout en prenant en compte une diversité de trajectoires militantes, d'espaces géographiques, politiques et culturels, ainsi que le point de vue de celles et ceux qui ont fréquenté ces organisations.

KAMGAIN, Olivia, M.A., administration publique, adjointe de recherche Université du Québec à Montréal

CHAMBERLAND, Line, Ph. D. Sociologie, professeure au département de sexologie Université du Québec à Montréal

LÉVY, Joseph J., Ph. D. anthropologie, professeur associé au département de sexologie Université du Québec à Montréal 


\section{RÉFÉRENCES}

Blais, M., J. Gervais et M. Hébert (2014). «Internalized homophobia as a partial mediator between homophobic bullying and self-esteem among youths of sexual minorities in Quebec », Ciencia e Saude Coletiva, vol. 19, $\mathrm{n}^{\circ} 2$, p. 727-735.

Cénat, J.-M., M. Blais, M. Hébert, F. Lavoie et M. Guerrier (2015). « Correlates of bullying in Quebec high school students: The vulnerability of sexual-minority youth », Journal of Affective Disorders, vol. 183, p. 315-321.

Chamberland, Line, Lévy, Joseph Josy, Kamgain, Olivia, Parvaresh, Pirayeh et Madeleine Bègue (2018), L'accès à l'égalité des personnes LGBT. Enjeux, luttes et alliances, dans Francine Saillant et Eve Lamoureux (dir.), InterReconnaissance. La mémoire des droits dans le milieu communautaire au Québec, Presses de l'Université Laval, p. 49 à 77.

Chamberland, L., et G. Richard (2017). «Changing the school climate: A critical examination of the policies and strategies against homophobia adopted in the province of Quebec (Canada) », dans S. T. Russell et S. Horn (dir.), Sexual Orientation, Gender Identity, and Schooling: The Nexus of Research Practice and Policy, Oxford, Oxford University Press, p. 194-215.

Chamberland, L., et M. Dorais (dir.) (2013). « L'homophobie, les tentatives de suicide et la résilience chez les jeunes LGBTQ », numéro thématique, Service social, vol. 59, n 1, p. 1-107.

Commission des droits de la personne et des droits de la jeunesse (2002). Jeunes gais et lesbiennes à l'école. Quels droits et libertés?, Montréal, Commission des droits de la personne et des droits de la jeunesse.

Conseil permanent de la jeunesse (2007). Sortir l'homophobie du placard... et de nos écoles secondaires - Recherche-avis, Québec, Conseil permanent de la jeunesse.

Coulombe, A. (2008). Lutte contre l'homophobie et pratiques d'empowerment auprès des lesbiennes, gais et bisexuel(les), maîtrise en travail social, Université du Québec à Montréal.

Demczuk, I., et F. Remiggi (dir.) (1998). Sortir de l'ombre. Histoires des communautés lesbienne et gaie de Montréal, Montréal, VLB éditeur.

Dorais, M. (2014a). Mort ou fif. Homophobie, intimidation et suicide, nouvelle édition revue et mise à jour, Montréal, Édition Typo.

Dorais, M. (2014b). De la honte à la fierté. 250 jeunes de la diversité sexuelle se révèlent, Montréal, VLB éditeur.

Dumas, J., J. J. Lévy, C. Thoër et B. Ryan (2010). « Minorités sexuelles et champ de régulation dans le cyberespace canadien : une étude exploratoire », dans P. Corriveau et V. Daoust (dir.), Régulation des minorités sexuelles, Québec, Presses de I'Université du Québec, p. 203-226. 
Enriquez, M. C. (2013). « La contestation des politiques de changement d'identité de genre par les militantes et militants trans québécois », Lien social et Politiques, n69, p. 181-196.

Enriquez, M. C. (2014). Le mouvement trans au Québec. Dynamique d'une militance émergente, Montréal, Cahier de l'IREF, coll. «Tremplin », nº 5 .

Groupe de travail mixte sur l'homophobie (2007). De l'égalité juridique à l'égalité sociale. Vers une stratégie nationale de lutte contre l'homophobie, Montréal, Commission des droits de la personne et des droits de la jeunesse. [www.cdpdj.qc.ca/publications/rapport homophobie.pdf]

Grundy, J., and M. Smith (2005). «The Politics of MultiscalarCitizenship: The Case of Lesbian and Gay Organizing in Canada.» Citizenship Studies, vol. 9, no. 4, pp. 389-404.

Laprade, B. (2013). « Le parallèle structurel entre la situation des jeunes LGBT et celle des organismes les desservant : quelques constats pour l'intervention », Service social, vol. 59, $\mathrm{n}^{\circ} 1$, p. 95-103.

Lépine, L., L. Chamberland, B. Carey et G. Bélanger (2017). Portrait des personnes LGBT+ en Gaspésie et aux Îles-de-la-Madeleine, Carleton-sur-Mer, Centre d'initiation à la recherche et d'aide au développement durable (CIRADD).

Lévy, J. J., et J. Dumas (2012). «Internet, santé et minorités sexuelles », dans C. Thoër et J. J. Lévy (dir.), Internet et santé. Acteurs, usages et appropriations, Québec, Presses de l'Université du Québec, p. 251-277.

Lévy, J. J., J. Dumas, B. Ryan et C. Thoër (dir.) (2011). Internet, santé et minorités sexuelles, Québec, Presses de l'Université du Québec.

Ministère de la Justice (2017). Plan d'action gouvernemental de lutte contre l'homophobie et la transphobie 2017-2022, Québec, Gouvernement du Québec. [www.justice.gouv.qc.ca/ministere/lalutte-contre-Ihomophobie]

Neveu, E. (2015). Sociologie des mouvements sociaux, Paris, La Découverte.

Pabion, L. (2016). Le processus de construction de l'identité collective du mouvement queer montréalais : perspectives militantes francophones, mémoire en science politique, Université de Montréal.

Paternotte, D. (2012). « La juridification ou le droit comme matrice de l'action collective : la revendication du droit au mariage entre personnes de même sexe », Politique et Sociétés, vol. 31, $n^{\circ} 2$, p. 93-112.

Richard, G., O. Vallerand, M.-P. Petit et A. Charbonneau (2015). « Discussing sexual orientation and gender in classrooms: A testimonial-based approach to fighting homophobia in schools », The Educational Forum, vol. 79, n 4, p. 421- 435. 
Saillant, F., et E. Lamoureux (dir.) (2018). InterReconnaissance. La mémoire des droits dans le milieu communautaire au Québec, Québec, Presses de l'Université Laval.

Secrétariat à la jeunesse (2017). Stratégie d'action jeunesse 2016-2021, Québec, Gouvernement du Québec. [www.jeunes.gouv.qc.ca/strategie]

Smith, M. (2011). "Canada: The power of institutions », dans M. Tremblay, D. Paternotte et C. Johnson (dir.), The Lesbian and Gay Movement and the State: Comparative Insights into a Transformed Relationship, Farnham, Ashgate, p. 73-88.

Tremblay, M. (2015). « Quebec and Sexual Diversity », dans M. Tremblay (dir.), Queer Mobilizations: Social Movement Activism and Canadian Public Policy, Vancouver, UBC Press, p. 106-124.

Veale, J., E. Saewyc, H. Frohard-Dourlent, S. Dobson, B. Clark et le groupe de recherche de l'enquête canadienne sur la santé des jeunes trans (2015). Être en sécurité, être soi-même. Résultat de l'enquête canadienne sur la santé des jeunes trans, Vancouver, Stigma and Resilience Among Vulnerable Youth Centre (Saravyc), Université de la Colombie-Britannique. 